\title{
The Effect of A Single Bout and Long Term HIIT on Muscular Cardiac and Circulatory System of Rats
}

\author{
Ryeaan A.M. Abdel Rahim
}

\author{
Specific Education Dept. Home Economics Nutrition, Port-Said Univ., Egypt \\ Received: 11 Nov. 2019 / Accepted 20 Dec. 2019 / Publication date: 30 Dec. 2019
}

\begin{abstract}
Aim of the study is to investigate: the effect of a single bout and long term high Intensity interval training on muscular, cardiac and circulatory system of rats. Materials and methods: 30 male Wistar Rats, weight 120-150g., Rats were divided into three groups of 10 rats each, control, single bout HIIT, and long term HIIT group, the latter was subjected to 4 weeks training period. Blood cells, Hemeoxygenase -1 in rat heart, T.cholesterol, TG, LDL, HDL, together with lactate and lactate dehydrogenase also VEGF and NO were determined results show a significant increased Rbcs, Hb and PCV in single bout and long term HIIT, also significant increased Hemeoxygenase-1 and HDL and significant decrease T.cholesterol , TG, LDL after HIIT. Also lactate and LDH significantly increased after single bout HIIT, and decreased in long term HIIT, VEGF and no significant increased after single bout and long term HIIT. With higher improvement after long term HIIT. Conclusion: The study has shown that a single bout and long term HIIT may have stimulating effect on blood cells, and increase $\mathrm{O}_{2}$ delivery, HIIT may support the role of Hemeoxygenase -1 as cardio protective protein and improve lipid profile, and influent muscle function together with the vasodilation of blood vessel and increasing blood flow to muscular, cardiac and vital organs.
\end{abstract}

Keywords: Single bout, long term HIIT, blood cells, Hemeoxygenase-1, lipid profile, VEGF.

\section{Introduction}

High-intensity interval training (HIIT) is a kind of physical exercise, it consists of successive repetitive high-intensity training with a period of relaxation, it is performed for shorter time and may result in more time-saving stimulation to increment aerobic capacity in skeletal muscle (Gibala et al., 2006; Burgomaster et al., 2008 and Terada et al., 2001) appeared that HIIT stimulates molecular transmission pathways connected to peroxisome proliferator activated receptor gamma co-activator-1 $\alpha$ (PGC-1 $\alpha$ ), which regulate mitochondrial biogenesis in humans and rodents (Soha, 2018) reported the benefits of HIIT, as it increase fat burning compare to other training as walking or jogging, it induce more fitness and endurance in a shorter time, also more strength to the skeletal muscles, in case of HIIT the body utilize $80 \%$ of the muscles compared to jogging which utilize $40 \%$ of muscle only, also HIIT help in reduce weight, and increase heart efficiency, and increase metabolism of the body and improve physical performance .

Mammals have a pronounced demand for oxygen. When environmental oxygen interchanges at the exterior of the lung alveoli, it is brought to the circulation, being disseminated to the tissue and to the terminal place for oxidative phosphorylation in the mitochondria of the cells. Skeletal muscle is distinctive in that it experiences considerable difference in oxygen tenseness $\left(\mathrm{PO}_{2}\right)$.

Calculations of myoglobin desaturation by $1 \mathrm{H}$ nuclear magnetic resonance spectroscopy $(1 \mathrm{H}$ NMRS) have determined variations in intracellular oxygen tenseness $\left(\mathrm{P}_{1} \mathrm{O}_{2}\right)$ ranging from values $<18$ $\mathrm{mmHg}$ at rest to values $>4 \mathrm{mmHg}$ through training (Richardson et al., 2001) to tolerate the massive increment in metabolic requirement, skeletal muscle tissue is depend on adequate oxygen provide to produce ATP from glucose and fatty acids.

Oxygen distribution is done by a complex system of systemic and local alterations in the cardiovascular system (Korthuis, 2011). Locally, incremented skeletal muscle blood influx is controlled via alterations in vascular tone (Snell and Mitchell, 1984), rearrangement of existing vessels in addition to the synthesis of novel vessels (angiogenesis) (Gute et al., 1996). During muscle contraction, arterial influx reduces as a reason of extravascular pressure extended on the vessels, however arterial influx increments as muscles rest between compressions. As a result, regular contractions leads to a pulsatile influx that participating to the functional hyperemia noticed in training (Clifford et al., 2006). 
The availability of oxygen can affect many signaling systems like the preference of fuel, synthesis of nitrogen oxide (NO) or reactive oxygen species (ROS), and the irritability of the membrane and $\mathrm{O}_{2}$ receptor systems (Clanton et al., 2013).

Hypoxia-inducible factor-1 (HIF-1) is a transcription factor that is expressed in response to physiological pertinent levels of oxygen deprivation (Jiang et al., 1996). Responses involve upregulation of genes with gene results that induce new vessel (Forsyth et al., 1996), red blood cell formation (Semenza, 1994), glucose transport (Glidel and Ratcliffe, 1997) and glycolysis (Firth et al., 1994), all of which are physiological adaptations of either increment oxygen supply or low oxygen consumption.

Oxygen is normal cellular function because it is a fundamental part of the electron transport chain for energy generation in cells. Any restriction in $\mathrm{O}_{2}$ accessibility incites a compensatory vasodilation directing blood flow upward to keep constant $\mathrm{O}_{2}$ delivery to the muscle.

It has been recommended that a more prominent proficiency of oxygen supply could enhance increment maximal performance in athletic, and it has been found that exercise performance was greatly reduced by extreme hypoxia and normoxia (Millek et al., 2012 and Rasmussen et al., 2010). The latter added that cerebral de-oxygenation precedes the development of central fatigue during exercise with both diminished cortical motor output and increased cerebral metabolism indicating that cerebral deoxygenation may confine performance (Amann et al., 2007) stated that a move from hypoxia to hyperoxia or norm at the end of exhausting hypoxic exercise supports the involvement of the central nervous system as a mediator of performance reductions under hypoxia.

Hypoxia or oxygen deprivation is induced when oxygen relating fails to meet the requirement of cells. This case is noticed in many pathophysiological conditions where blood influx reduces or the request of oxygen grants relatively increments in tumor genesis, also in physiological situation like muscle training or in high altitude, or a severe localized ischemia. Cellular hypoxia induces a wide range of physiological and patho-physiological responses in an organism at the systemic and cellular levels (Fedele et al., 2002). The responses include: increased ventilation and cardiac output, a switch from aerobic to anaerobic metabolism, promotion of improved vascularization, through the activation of proteins or mediated by hypoxia-inducible factor-1 (HIF-1) (Semenza, 2000).

There are many affirmations that exercising in oxygen deficit impacts muscular functions, and an overall number of genes interfere with hypoxia inducible factors (HIFs) (Semenza et al., 2006), HIF (1) is well known to be a basic factor for stimulating many genes assisting cell viability like vascular endothelial growth factor, endothelin 1, heme-oxygenase -1 , glucose metabolism and anemia control (transferrin and erythropoietin (Rosenberger et al., 2002).

Hypoxia may be also accompanied by increment the expression of inducible nitric oxide synthase (iNOS), where iNOS was found to be up-regulated in heart in vivo and after ischemia (Grilli et al., 2003).

Aim of the present study is to investigate the effect of a single bout and long term HIIT on muscular cardiac and circulatory system of rats.

\section{Materials and Methods}

\subsection{Animals and Experimental design}

A total of 30 healthy male Wistar Rats weight between 120-150g. Rats were housed in individual stainless steel cages, provided with food and water ad libitum.

Rats were residents in animal room which preserved on a $12 \mathrm{~h}$ light-dark cycle at an ambient temperature of $25 \pm 2$ and $35-50 \%$ humidity for two weeks prior beginning the investigation for adaptation. Rats were distributed into three groups, the normoxic group $(\mathrm{n}=10)$ and the exercise groups, whether a single bout of high intensity interval training (HIIT) $(n=10)$, long term HIIT $(n=10)$ for 4 weeks using some modification than HIIT protocol described by Terada et al. (2005). Rats achieved a 20 s swimming training at most 20 times or until reach exhaustion, with weight equivalent to $10 \%$ of their body weight, tied near the base of their tail, in a glass container filled with water.

Exhaustion was reached as when rat could not rise to surface more than 3s. During the training periods, rats were lowered from the water, and then rats were allowed to rest for 10 seconds.

Water temperature during the exercise swim is set at $35^{\circ} \mathrm{C}$. 
Long term HIIT was for 4weeks period/once per day in the morning (9:00 AM to 11:00 AM) for 5 days/week. The experimental protocol used was approved by the laboratory animal control guidelines at Port-Said University.

\subsection{Blood and tissues samples}

At the end of the experiments, Rats were anaesthetized immediately by ether, blood was withdrawn by cardiac puncture collected into sterile test tubes containing EDTA for blood picture analysis by coulter Counter, lactate was determined by accusport, plasma nitrite was determined by Moshage et al. (1995) Method.

LDH level using the procedure of Streovan and Makaroval (1989), VEGF using Elisa method He et al. (1989) method. T. Cholesterol using Allain et al. (1974) method.

Trighly cerides was estimated by McGowan et al., 1983), HDL, LDL according to Wieland and Seidel (1983). After sacrifying the rats, Heme oxygenase-1 (Ho-1) was determined in heart tissue extract prepared as described by Maines (1988) for Ho-1 assay by quantitative sandwich immunoassay (Kitchin et al., 2001), Elisa technique.

\subsection{Statistical Analysis}

Results were analyzed with the statistical package for the social sciences (SPSS) version 10 and presented as mean \pm standard error of mean. Unpaired " $t$ " test was utilized to evaluate the difference between groups and one-way Anova (F-Test).

Variations among groups were theorized $\mathrm{d}$ to be significant at $\mathrm{P}>0.05$.

\section{Results}

Table 1: Effect of single bout of HIIT and long term of HIIT on some blood picture

\begin{tabular}{lccc}
\hline Parameter & Control & Single bout HIIT & Long term HIIT \\
\hline Wbc $\left(\mathbf{T h o} / \mathbf{m}^{3}\right)$ & $7.3 \pm 0.4$ & $7.4 \pm 0.5$ & $7.3 \pm 0.6$ \\
Rbcs $\left(\mathbf{M i l} / \mathbf{m}^{3}\right)$ & $6.1 \pm 0.3$ & $6.3 \pm 0.5$ & $7.2 \pm 0.4 *$ \\
Hemoglobin g/dl & $10.5 \pm 0.2$ & $11.3 \pm 0.4$ & $13.8 \pm 0.6 *$ \\
PCV \% & $37.4 \pm 1.3$ & $40.2 \pm 1.7$ & $43.5 \pm 1.5 *$ \\
\hline
\end{tabular}

Mean \pm SEM $n=10$ of each group

Significant $\mathrm{P}<0.05$

Non-significant change of wbcs was recorded Rbcs, Hb, PCV significantly increased post single bout and long term HIIT, another elevation noticed post long term HIIT compared to control.

Table 2: Effect of single bout of HIIT and long term of HIIT on Heme oxygenase -1 in heart tissue, cholesterol and triglycerides, HDL and LDL.

\begin{tabular}{lccc}
\hline Parameter & Control & Single bout HIIT & Long term HIIT \\
\hline Heme Oxygenase ug/mg protein & $0.6 \pm 0.11$ & $1.52 \pm 0.14$ & $1.76 \pm 0.13$ \\
Total cholesterol mg/dl & $49.6 \pm 1.5$ & $41.2 \pm 1.2$ & $36.8 \pm 0.9$ \\
Triglycerides mg/dl & $126 \pm 4.3$ & $112.1 \pm 3.8$ & $104.2 \pm 3.2$ \\
HDL mg/dl & $26.4 \pm 2.1$ & $28.7 \pm 2.6$ & $32.6 \pm 2.9$ \\
LDL $\mathbf{~ m g / d l ~}$ & $18.7 \pm 1.3$ & $16.2 \pm 1.1$ & $15.3 \pm 1.2$ \\
\hline
\end{tabular}

Significant $\mathrm{P}<0.05$

Heme oxygenase-1, HDL significantly increased post single bout and long term HIIT, more improvement shown by long term HIIT, while T.cholesterol, TG significantly decreased post single bout and long term HIIT, improvement shown by long term HIIT compare to single bout and control group.

Table 3: Effect of single bout of HIIT and long term HIIT on lactate and LDH levels

\begin{tabular}{lccc}
\hline Parameter & Control & Single bout HIIT & Long term HIIT \\
\hline Lactate (mmol/l) & $0.86 \pm 0.2$ & $2.5 \pm 0.8^{*}$ & $0.82 \pm 0.1^{*}$ \\
LDH (IU/L) & $69.1 \pm 9.3$ & $88 \pm 10.4^{*}$ & $63.2 \pm 6.5^{*}$ \\
\hline
\end{tabular}

Mean \pm SEM $n=10$ of each group

Significant $\mathrm{P}<0.05$

Lactate and LDH significantly increased post single bout of HIIT, while long term HIIT showed significant decreased of lactate and LDH compared to single bout HIIT and control groups. 
Table 4: Effect of single bout of HIIT, and long term HIIT on (VEGF) vasoendothelial growth factor and plasma nitrite

\begin{tabular}{llll}
\hline Parameter & Control & Single bout HIIT & Long term HIIT \\
\hline VEGF Pg/ml & $35.3 \pm 6.34$ & $49.3 \pm 7.6^{*}$ & $80.2 \pm 10.4^{*}$ \\
Plasma Nitrite (umol/L) & $47 \pm 11.2$ & $57.4 \pm 9.6^{*}$ & $76.3 \pm 11.5^{*}$ \\
\hline
\end{tabular}

Mean \pm SEM $n=10$ of each group

Significant $\mathrm{P}<0.05$

VEGF and plasma Nitrite show significant increased post single bout of HIIT and long term HIIT, the Latter show another significant increased concentration compared to single bout of HIIT and control groups.

\section{Discussion}

High intensity interval training (HIIT) is the exchange between cardio training and high intensity training, meaning between high intensity and low intensity training, as example beginning with low intensity for a minute than with high intensity for 30s than repeating exercise training for $10 \mathrm{~min}$., the HIIT must begin with warm up $5 \mathrm{~min}$. and end with cooling down for $4 \mathrm{~min}$. and the whole training may reach 20-25 min. this may be used also for laboratory investigations. Researchers have used HIIT in human and animals (Soha, 2018 and Terada et al., 2005).

Table (1) shows the impact of one bout of HIIT and long term of HIIT on some blood picture of rats, revealed a beneficial effect on the hemopoietic system, as indicated from the significant increase of Rbcs count, $\mathrm{Hb}, \mathrm{PCV}$, of single bout and long term effect of HIIT induced a higher level of improvement in all parameters determination, which indicated that long term exercise induced a higher adaptation of the hemopoietic system. Due to stimulation of stem cells as a result of stress induced training which in turn increased stem cells release and blood cells and component formation. At the same direction, Mohamed and Saleh (2015) reported a significant increase in Rbcs count, $\mathrm{Hb}$ concentration and PCV after sport diving.

This result is in accordance with that of Soha (2018) and Schnerman (2002) that exercise training induce many benefits such as increase in muscle and heart efficiency and the hemopoietic system which increased blood flow and availability of $\mathrm{O}_{2}$ supply which in turn increase metabolism of the body and improve physical performance. Guyton and Hall (2006) added that training specially regular exercise induce many benefits to the different organs of the body as it increase blood gases and oxygen diffusing capacity of athletes, example oxygen diffusing capacity increased from $23 \mathrm{ml} / \mathrm{min}$. in non-athlete at rest to $71 \mathrm{ml} / \mathrm{min}$. in swimmers during maximal exercise. Together with improve of cardio vascular system and respiratory system in exercise.

The hemoglobin is destroyed in the blood, and the globin portion is reutilized and the iron of haem enters the iron pool, for rescue, as for porphyrim of haem is degraded in the reticulo endothelial cells of the liver spleen and bone marrow. Haem catabolism is achieved in the microsomal fractions of the reticulo endothelial cells by a complex enzyme system known as haem oxygenase (Murray et al., 2009). In table (2) heme oxygenase-1 (Ho-1) showed increased level in myocardial tissue of rats subjected to single bout and long term HIIT. The increased (Ho-1) related to exercise training supports its role as cardio protective proteins.

Nishikawa et al. (2004) reported that carbon monoxide, Ho-1 and Nitric oxide (NO) can participate in vascular tone regulation during normal and abnormal physiological conditions including hypoxia.

Table $(1,2)$ represent the effect of single bout of HIIT and long term of HIIT on Hemoglobin and heme oxygenase-1, there was a significant increased hemoglobin concentration and heme oxygenase-1 indicating that intensive exercise on rats may induce hypoxia and at the same time stimulate action of hypoxia inducible factor-1 genes related to the muscle action, heart function and blood vessels and energy metabolism of the cells.

Semenza (2010) reported that under oxygen deficit situations, the energy metabolism of the cells shifts from aerobic to anaerobic, and HIF-1 $\alpha$ regards to this shifts through glycogen metabolism Gene transcription. Mougios (2005) stated that blood hemoglobin concentration increases at high altitude, as $\left(\mathrm{O}_{2}\right)$ in the air drops, the same happen in the blood, this in turn stimulate erythropoietin release by the kidneys causing erythrocyte formation and in the same time hemoglobin, He also added that the amount of hemoglobin increase during exercise as water exits the blood vessel to the sweat, leading to hemo concentration which may last for 1hour after exercise. Also PCV increase as it is the volume of erythrocyte as a percentage of the blood volume. 
Cholesterol is an amphipathic lipid and is a crucial structure constituent of membranes and the exterior layer of plasma lipoproteins. Cholesteryl ester is a storage form of cholesterol presented in nearly all tissues. It is imparted in the core of lipoproteins. LDL is the precursor of cholesterol and cholesteryl ester uptake into many tissues free cholesterol is eliminated from tissues by HDL and imparted to the liver for transformation to bile acids. Cholesterol is as a factor in the genesis of atherosclerosis causing vascular disease (Chatterjea and Shinde, 2005) lifestyle modification is the preferable form of treatment for most types of hyperlipidemia (National Cholesterol Education Program, 1993).

Table (2) illustrated the effect of single bout of HIIT and long term HIIT on total cholesterol (TC), HDL-C, LDL-C, and triglycerides, TC, LDL-C and triglycerides showed significant decrease compared to control rats, while HDL-C showed a significant increase compared to control rats. Long term HIIT induced a higher level of HDL-C and lower level of TC, LDL-C, triglyceride compared with a single bout of HIIT.

These results were in accordance with those of Soha (2018), (Shaw et al., 2006), some researchers determined the effects of exercise on lipogram TC, TG, LDL and HDL and reported an improvement of all parameters studied (Ihab, 2017; Comcepcion et al., 2006 and Kent, 2011), they concluded that there was a significant decrease affecting HDL which affect the vascular system and the health positively with decreased weight following exercise program, it is also well noted that exercise alone is not sufficient for health benefit, this must induced in relation to a balanced nutritional program for optimal results.

Table (3) shows the effect of a single bout of HIIT and long term HIIT on lactate and lactate dehydrogenase enzyme level on rat, there was a significant rise of lactate and LDH following single bout of HIIT compared to control group, while long term HIIT significantly decreased lactate, LDH.

This is in accordance with Galal (2017 and Laurent et al. (2007). The possible cause of the elevated lactate and LDH following single bout of HIIT may be due to the anaerobic glycolysis leading to the conversion of pyruvic acid to lactic acid the reaction takes place in the cytosol and is catalyzed by lactate dehydrogenase enzyme, the elevated lactate concentration during maximal exercise indicating a massive anaerobic catabolism of carbohydrate.

Mougios (2006) reported that LDH is a tetramer composed of two kinds of similar subunits denoted by $\mathrm{H}$ for heart and $\mathrm{M}$ for muscle and the enzyme help in the conversion of pyruvate and lactate in two directions and the increase in lactate concentration is the most impressive change in the concentration of a metabolite during exercise, as lactate can crosses membranes easily and enter in skeletal muscles that did not participate in the exercise also the heart and kidneys and can transverse from white muscle to red muscle fibers to be converted to energy in the mitochondria, in this way lactate may be used in energy production.

Laurent et al. (2007) added that the liberation of lactate and $\mathrm{H}+$ ions to the exterior of the muscle cells component a protective mechanism versus intra cellular $\mathrm{pH}$ lowering and lactate accumulation, this is mediated by lactate-H+ transport through MCT4, this predominates through acute training, thus the muscle contents of MCT1 and MCT4 influence work capacity during high intensity exercise. In case of the effect of long term HIIT, the significant decreased of lactate and LDH may be due to adaptation to exercise, post long term HIIT, the adaptation process was due to increase number and size of Mitochondria following long term exercise which may increase energy production and $\mathrm{O}_{2}$ availability (Mougios, 2006).

Current indication that mitochondria biosynthesis in skeletal muscle is controlled partially via a Redox-sensitive mechanism and supported by Holloszy (2008), stated that in high intensity exercise increase production of ROS through xanthine oxidase (xo), stimulate PGC $1 \alpha$ expression and NRF-1 and transcription factor A. this signaling event is concomitant with stimulation of P38MAPK and CAMP reaction element binding protein (CREB) phosphorylation.

Inhibition of (XO) by allopurinol hardly impaired exercise stimulation of PGC- $1 \alpha$ signaling pathway. So, mitochondrial biogenesis may in turn increase $\mathrm{O}_{2}$ availability and affect lactate production and decrease sign of fatigue.

Table (4) shows a significant increased nitrite (NO) as compared to control rat, the increase level of NO due to a single bout of HIIT indicated that high intensity interval training may induce a positive effect in vasodilatation of blood vessel which in turn help in increasing oxygen supply to important 
organs of the body, long term of HIIT induced another increased level of NO and an elevation of oxygen supply to tissues.

The effect of exercise as an indicator of angiogenesis and oxygen supplier to organs and tissues was also reported by researchers Convay et al. (2001); Arany et al. (2008)and Ke and Costa (2006).

The role of HIIT in Table (4) shows an elevated level of VEGF due to the training of swimming rats leading to angiogenesis following single bout of HIIT, another elevation of VEGF was also shown following long term HIIT compared to control group suggesting that high intensity inducible training is associated with angiogenesis, which affect organs of the body that help in health and fitness of the subject, this was also reported by Ohno et al. (2012) and Manalo et al. (2005).

Moreover, (Ohno et al., 2012) have shown that severe training indicated that various constituents of the HIF-1 pathway, including VEGF and erythropoietin are stimulated concerning change in oxygen request in muscles, and that endurance training appears to improve muscle $\mathrm{O}_{2}$ transport and metabolism. Also inducing increments VEGF expression and angiogenesis this suggests that HIF-1 $\alpha$ distinct controlling of VEGF and angiogenesis.

This summarizes the considerable of some situation, such as oxygen deficit, oxidative stress and training in HIF-1 and VEGF signaling pathway in skeletal muscle.

In conclusion, the present study has shown the influence that a single bout of HIIT and long term HIIT may have in stimulating Rbcs, $\mathrm{Hb}, \mathrm{PCV}$ in increasing $\mathrm{O}_{2}$ delivery to muscle and heart and vital organs. More specifically, to underline the role of HIIT may play to support the action of Heme oxigenase-1 as cardio protective protein together with improve lipid profile (T. cholesterol, TG, LDL and HDL). The influence of single bout and long term HIIT on lactate and LDH in blood and muscle function has also been shown and that long term HIIT elevated muscle fitness and decreased fatigue.

At last, the present results show the role played by VEGF NO in vasodilation and increasing blood flow to tissues and organs of the body.

\section{References}

Allain, C., L. Poon, and C. Chan, 1974.Enzymatic determination of total serum cholesterol Clin. Chem., 20:470.

Amann, M., M. Romer, and J. Dempsey, 2007. Severity of arterial hypoxemia affects the relative contribution of peripheral muscle fatigue to exercise performance in healthy humans. J. physiol., $581: 389$.

Arany, Z., S. Foo, and Y. Ma, 2008. HIF independent regulation of VEGF and angiogenesis by Transcriptional co-activator PGC-1 $\alpha$ Nature, 451: 1008.

Burgomaster, K., K. Howarth, and M. Gibala, 2008. Similar metabolic adaption during exercise after low volume script Interval and traditional endurance training in humans. J physiol., 586:160.

ChatterJea, M., and R. Shinde, 2005. Text book of Medical Biochemistry JAYPEE, India.

Clanton, T., M. Hogan, and L. Gladden, 2013. Regulation of cellular gas exchange, oxygen sensing and metabolic control. Compre. Physiol., 3, 1135.

Cliff Ford, P., H. Kluess, and J. Hamann, 2006. Mechanical compression elicits vasodilatation in rat skeletal Muscle feed arteries. J. Physiol., 572:561.

Concepcion, A., G. Mercedes and G. Angel, 2008. Alterations in plasma and tissue lipids associated with obesity and metabolic syndrome Clinical science, 114:183.

Convay, E., D. Collen, and P. Cameliet, 2001. Molecular mechanism of blood vessel growth cardio Vascular Res., 49:507.

Fedele, A., M. White Law, and D. Peet, 2002. Regulation of gene expression by the hypoxia inducible factors Mol. Interv., 2,229.

Firth, J., B. Ebert, and C. Pugh, 1994. Oxygen related control elements in the phosphor-glycerate kinase and lactate dehydrogenase. PROC NATL Acad. Sci., 91, 6496.

Forsythe, J., B. Jiang, and N. Lyer, 1996. Activation of VEGF gene transcription by $\mathrm{HIF}_{1}$ Mol Cell Biol., 16, 4604.

Galal, A., 2017. Diversity of ACE and Actinin 3 and its relation to biological Variables in aerobic and anaerobic athletes. PHD, Fac. of Physiol Education, Assiot. Univ.

Gibala, M., J. Little, and S. Raha, 2006. Short term sprint interval versus traditional endurance training J. physiol., 575:901. 
Gleadle, J., and P. Ratcliffe, 1997. Induction of HIF1, erythropoietin, VEGF, and glucose transporter by hypoxia blood, 89,503.

Grilli, A., M. De Lutiis, and A. Patruno, 2003. Inducible NO synthase and heme oxygenase1 in rat heart. Ann. Clin. Lab. Sci., 33, 208.

Gute, D., C. Fraga, and J. Amann, 1996. Regional changes in capillary supply in skeletal muscle of high intensity endurance trained rats. JAPPL. Physiol., 81, 619.

Guyton, A. and J. Hall, 2006. Text book of medical physiology. El Sevier Sanders, USA. Hatfield, F., 2013. Fitness: the complete guide. ISSA, USA.

He, H., V. Venema, and X. Gu, 1999. Vascular endothelial growth factor signals endothelial Cell production of nitric acid and prostacyclin through FLK-1/KDR activation of C-SRC. J. Biol. Chem., 274, 25130.

Holloszy, J., 2008. Regulation by exercise of skeletal muscle content of mitochondria and GLUT4. J physiol. Pharmacol., 59,5.

Ihab, M., 2017. Effect of resistance training on some biochemical variables. PHD thesis, Monufia Univ.

Jiang, B., G. Semenza, and C. Baner, 1996. $\mathrm{HIF}_{1}$ levels vary exponentially over a physiologically relevant Range of $\mathrm{O}_{2}$ tension Am J physiol., 271, 1172.

Ke, Q., and M. Costa, 2006. Hypoxia inducible factor-1 Mol. Pharmacol., 70:1469.

Kemt, H., 2011. Long term weight loss Et updated now, 17.

Kitchin, K., W. Anderson, and M. Sue Matsu, 2001. An Elisa assay for heme oxygenase (Ho.1) Immunol methods, 247, 153.

Korthnis, R., 2011. In skeletal muscle Circulation, San, Rafael, (CA).

Laurent, M, M. Kristensen, and C. Denis, 2007. Importance of PH regulation and lactate $\mathrm{H}^{\text {and }}$ transport Capacity for work production during supraximal exercise in humans. J. APPL. Physiol., 102, 1936.

Maines, M., 1988. Heme oxygenase function multiplicity, regulatory mechanism and clinical applications FASEBJ, 10:2557.

Manalo, D., A. Rowan, and T. Lavoie, 2005. Transcription regulation of VEGF responses to hypoxia by HIF-1 J. blood, 105: 659.

Mcgowan, M., J. Artiss, and B. Zak, 1983. A peroxidase coupled method for the colorimetric determination of striglycerides. Clin. Chem., 29: 538.

Millet, G., M. Muthalib, and K. Nosaka, 2012. Severe hypoxia affects exercise performance independently of afferent feedback and peripheral fatigue. J. APPL. Physiol., 11:1335.

Mohamed, S., and T. Saleh, 2015. The effect of sport diving on stem cells CD34 and and complete blood picture. The Swedish J of scientific research, 2:45.

Moshage, H., B. Kok, and L. Jansen, 1995. Nitrite determination in plasma Clin. Chem. 41: 892.

Mougios, V., 2006. Biochemistry of exercise Human kinetics, USA.

Murray, R., D. Benfer, and P. Weil, 2009. Harper illustrated biochemistry. Lange, MSA.

National cholesterol Education Program, 1993. Second report of the expert panel on detection, Evaluation and treatment of high blood cholesterol in adults. National Institutes of Health Publ. No. 93:3095 US.

Nishikawa, Y., D. Stepp, and D. Merkus, 2004. In vivo role of heme oxygenase in is chemic coronary vasodilatation Am J. physiol. Heart Cir. Physiol., 286: 2296.

Ohno, H., Shiratok, and T. Sakurai, 2012. Effect of exercise on HIF-1 and VEGF signaling. J phys. Fitness sports Med., 1, 5.

Rasmussen, P., J. Nielsen, and N. Petersen, 2010. Reduced muscle activation during exercise related to brain Oxygenation and metabolism in humans. J. physiol., 588:1985.

Richardson, R., S. Newcomer, and A. Noyszewski, 2001. Skeletal muscle intracellular PO assessed by myoglobin desaturation. J. physiol., 91:2679.

Rosenberger, C., S. Mandriota, and S. Jurgensen, 2002. Expression of HIF1 $\alpha$ and 2 alpha in hypoxia and ischemic rat kidney. J. Am. Soc. Nephrol., 13,1721.

Schnerman, J., 2002. Exercise. Am J physiol. Regul. Integr comp. 2,283.

Semenza, G., 2010. HIF-1 upstream and downstream of cancer metabolism. Curr. Opin. genet. Dev., 20:51.

Semenza, G., Shimoda, L., and N. Prabbakar, 2006. Regulation of gene expression by $\mathrm{HIF}_{(1)}$ Novartis foundation symp., $272,2$. 
Semenza, G., 2000. Oxygen regulated transcription factors and their role in Pulmonary diseases. Respire. Res., 1:159.

Semenza, G., 1994. Regulation of erythropoietin production. Hematol clin. North Am. 8,863. Shaw, K, H. Gennet, and P. Rourke, 2006. Exercise, overweight or obesity. John Willey, Sons.

Snell, P., and J. Mitchell, 1984. The role of maximal oxygen uptake in exercise performance Clin. chest Med., 5,51.

Soha, A., 2018. The role of metabolic gene in weight loss by using nutritional and athletic programs. $\mathrm{PhD}$ thesis, Fac. physical Education, Sadat Univ.

Streov, E., and V. Makarova, 1989. Photo color metric method for estimating LDH in blood serum. Laboratory Manual in biochemistry Mir publishers, Moscow, Russia, 106.

Terada, S., K. Kawanaka, and M. Goto, 2005. Effects of high intensity intermittent swimming on PGC- 1 Alpha protein expression in rats skeletal muscle. Acta. Physiol. Scand, 184,59.

Wieland and Seidel, 1983. A simple specific method for prediction of low density lipoproteins. J Lipid research, 24, 904. 